Musées, Patrimoine et Culture scientifiques et techniques

$108 \mid 2006$

novembre-décembre 2006

\title{
20 ans de Planétariums
}

\section{Agnès Acker}

URL : http://journals.openedition.org/ocim/1314

DOI : $10.4000 /$ ocim. 1314

ISSN : 2108-646X

Éditeur

OCIM

Édition imprimée

Date de publication : 25 novembre 2006

ISSN : 0994-1908

\section{Référence électronique}

Agnès Acker, « 20 ans de Planétariums », La Lettre de l'OCIM [En ligne], 108 | 2006, mis en ligne le 12 mars 2014, consulté le 01 mai 2019. URL : http://journals.openedition.org/ocim/1314 ; DOI : 10.4000/ ocim. 1314 


\section{0 ans de planétariums}

Agnès Acker *

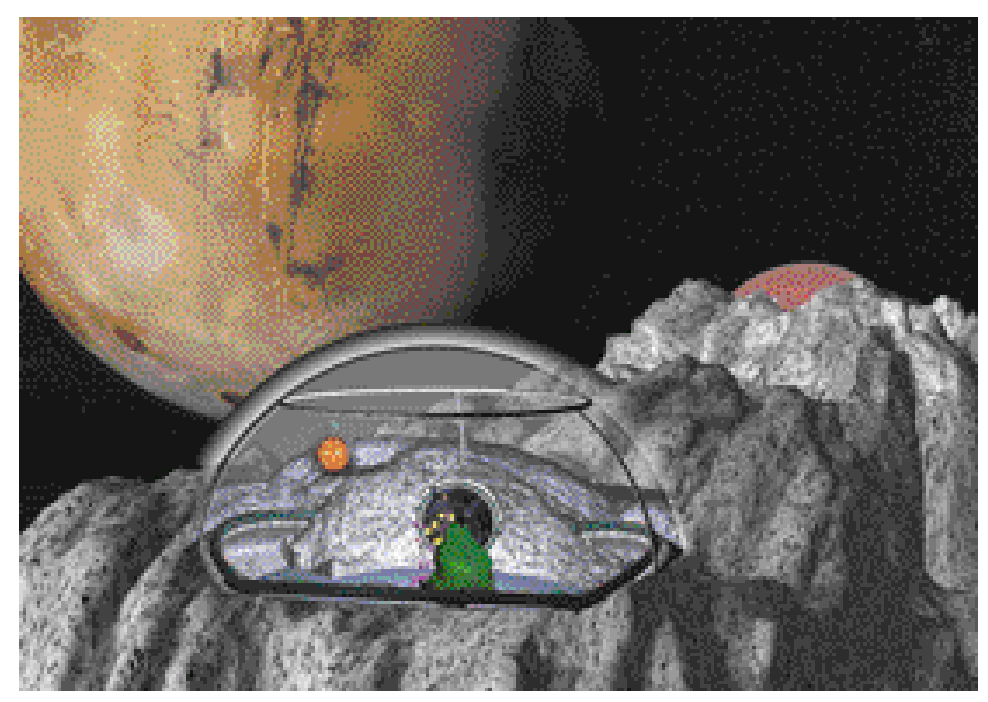

Planètes à l'horizon, spectacle co-produit par les planétariums du Forum départemental des Sciences à Villeneuve d'Asca et du Palais de l'Univers à Cappelle-la-Grande () Violette Le Quéré-Cady

* Agnès Acker est astrophysicienne, professeur à l'université Louis Pasteur de Strasbourg et présidente de l'association des planétariums

de langue française

Observatoire de Strasbourg

11 rue de l'Université 67000 Strasbourg téléphone + 33390242467 acker@astro.u-strasbg.fr
Face à l'accroissement du nombre des planétariums enregistré en France depuis une vingtaine d'années, l'auteur montre la grande diversité actuelle de ces outils efficaces de diffusion de la culture scientifique et technique, I'apport récent de la technologie numérique et son importance dans l'attractivité des nouveaux spectacles proposés par ces établissements ainsi que l'évolution vers une plus grande professionnalisation des métiers et notamment la place essentielle tenue par les animateurs de planétariums.

En 1980, deux planétariums publics étaient implantés en France. En 2006, 26 planétariums fixes fonctionnent dans des structures dédiées à la culture scientifique et technique ou dans des bâtiments publics. De plus, une centaine de petites structures sont répertoriées, fixes ou itinérantes dans les départements et les régions. Les techniques de projection ont évolué et se sont complexifiées, ainsi que les séances de planétarium et les expertises des animateurs et responsables.

\section{L'association des planétariums de langue française}

Depuis sa création il y a 20 ans, l'association des planétariums de langue française (APLF) œuvre pour développer le monde des planétariums, tout particulièrement en France. Il serait souhaitable que 


\section{Les Planétariums en France}

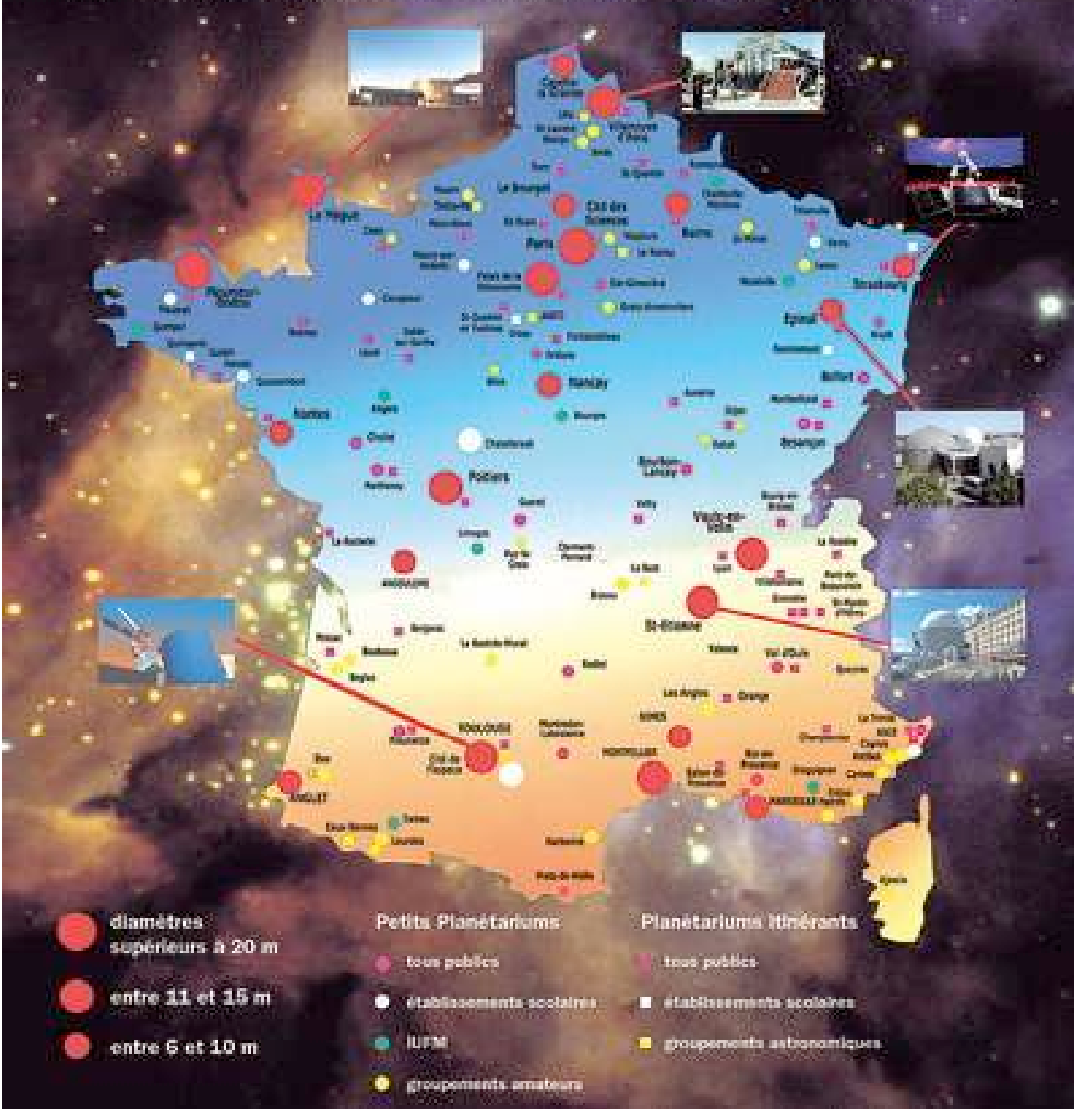

Répartition des planétariums en France au début 2005 ๑) APLF

chaque année un nouveau planétarium voit le jour, de sorte que toutes les villes françaises de plus de 10000 habitants se trouvent enrichies d'un tel outil pédagogique et culturel.

L'APLF réunit environ 110 membres actifs, œuvrant essentiellement dans des planétariums français, mais aussi à Brescia (Italie), Bruxelles et Genk (Belgique), Lucerne (Suisse), Barcelone (Espagne), Montréal (Canada), Lafayette et Louisiane (États-Unis), Tunis (Tunisie), Alger (Algérie), Rabat (Maroc)...

Les activités de l'APLF se déroulent autour de trois missions :

- partager les savoirs et savoir-faire, à travers les colloques et la revue Planétariums annuels, et un site Internet constamment actualisé www.aplf-planetariums.org ;
- réaliser en commun des documents pédagogiques (livrets, cédéroms - voir la Lettre de l'OCIM n ${ }^{\circ} 103$ ) et produire/échanger des spectacles de planétariums pour la communauté ;

- organiser des actions de formation (en 1990-1991, 1994, 1999, 2003, 2005-2007).

Une interaction régulière est engagée avec l'international planetariums society (IPS), avec partage d'une vision toujours remise en question de la place des planétariums dans la culture et l'éducation.

L’APLF est hébergée par l'observatoire de Strasbourg, et depuis 2001 bénéficie d'un emploi permanent (emploi-jeune consolidé). 


\begin{tabular}{|c|c|c|c|c|c|c|}
\hline \multirow[t]{2}{*}{ Ville } & \multirow{2}{*}{$\begin{array}{l}\text { Type de } \\
\text { gestion }\end{array}$} & \multirow{2}{*}{$\begin{array}{l}\text { Diamètre } \\
(\mathrm{m})\end{array}$} & \multicolumn{2}{|c|}{ Équipement à l'ouverture } & \multicolumn{2}{|c|}{ Renouvellement } \\
\hline & & & année & système & année & système actuel \\
\hline $\begin{array}{l}\text { Paris, Cité des Sciences } \\
\text { et de l'Industrie }\end{array}$ & $\mathrm{C}$ & 21 & 1986 & $\mathrm{OM}(\mathrm{S})$ & 2006 & N (6 VP - RSA) \\
\hline Pleumeur-Bodou & A & 20 & 1988 & OM $(Z)$ & 1998 & $\mathrm{~N}(6 \mathrm{VP})$ \\
\hline Paris, Palais Découverte & $\mathrm{C}$ & 15 & 1937 & OM (Z) & 1997 & OM (Z) \\
\hline Toulouse & $\mathrm{C}$ & 15 & 1997 & $\begin{array}{l}\mathrm{N} \text { (fisheye } \\
\text { vectoriel - D) }\end{array}$ & 2005 & N (2X6 VP - D) \\
\hline Vaulx-en-Velin & $a-p$ & 15 & 1995 & $\begin{array}{l}\mathrm{N} \text { (fisheye } \\
\text { vectoriel - D) }\end{array}$ & 2006 & $\mathrm{~N}(6 \mathrm{VP}-\mathrm{D})$ \\
\hline Montpellier & $\mathrm{a}-\mathrm{p}$ & 15 & 2002 & $\begin{array}{l}\mathrm{N} \text { (fisheye } \\
\text { vectoriel - D) }\end{array}$ & & $\mathrm{N}(6 \mathrm{VP}-\mathrm{D})$ \\
\hline Rennes & $\mathrm{C}$ & 14 & 2006 & $\mathrm{~N}(6 \mathrm{VP}-\mathrm{SS})$ & & \\
\hline Villeneuve d'Ascq & $\mathrm{C}$ & 14 & 1996 & OM (RSA) & & \\
\hline Poitiers & $\mathrm{C}$ & 12 & 1992 & $\mathrm{OM}(\mathrm{S})$ & & \\
\hline Saint-Étienne & $\mathrm{a}-\mathrm{a}$ & 12 & 1997 & OM (RSA) & 2002 & $\mathrm{~N}(6 \mathrm{VP}-\mathrm{RSA})$ \\
\hline La Hague & a-p & 10 & 1999 & $\mathrm{OM}(\mathrm{Z})$ & & \\
\hline Épinal & $\mathrm{a}$ & 10 & 2002 & OM (RSA) & & \\
\hline Dijon & $\mathrm{C}$ & 10 & 2005 & $\mathrm{~N}(\mathrm{RSA})$ & & \\
\hline Cappelle-la-Grande & $\mathrm{C}$ & 9 & 1989 & OM (RSA) & & \\
\hline Le Bourget & a-p & 8,2 & 1984 & OM (Z) & 2000 & OM (RSA) \\
\hline Nantes & a-p & 8 & 1981 & OM (Z) & 2005 & $\mathrm{~N}(5 \mathrm{VP}-\mathrm{RSA})$ \\
\hline Nîmes & $a-p$ & 8 & 1982 & $\mathrm{OM}(\mathrm{Z})$ & & \\
\hline Strasbourg & U & 8 & 1982 & $\mathrm{OM}(\mathrm{S})$ & & \\
\hline Nançay & $\mathrm{a}-\mathrm{a}$ & 7 & 1997 & OM (RSA) & & \\
\hline Reims & a-p & 6 & 1979 & $\mathrm{OM}(\mathrm{Z})$ & & \\
\hline Châtellerault & $\mathrm{a}$ & 6 & 1991 & OM (jide) & & \\
\hline Marseille & $\mathrm{a}-\mathrm{a}$ & 6 & 2001 & OM (RSA) & 2005 & $\mathrm{~N}$ (fisheye coul d) \\
\hline Montredon-Labessonie & $\mathrm{a}-\mathrm{a}$ & 5,4 & 1993 & $\mathrm{OM}(\mathrm{G})$ & & \\
\hline Aix-en-Provence & $\mathrm{a}-\mathrm{a}$ & 5 & 2002 & OM (RSA) & & \\
\hline Belfort & $\mathrm{a}-\mathrm{a}$ & 5 & 1982 & $\mathrm{OM}(\mathrm{G})$ & & \\
\hline Bourbon-Lancy & $\mathrm{a}-\mathrm{a}$ & 5 & 1993 & $\mathrm{OM}(\mathrm{G})$ & & \\
\hline
\end{tabular}

Les planétariums fixes en France (d'après Audéon \& Ruiz, Planétariums, 2006)

Gestion : $\mathrm{C}=$ lieu de CSTI, $\mathrm{U}=$ université, $a=$ autonome, $a-a=$ association ou SEM, $a-p=$ gestion par collectivité

Système : $O M=$ optomécanique, $N=$ numérique

Technique : Z = Zeiss, $S=$ Spitz, $S S=$ SkySkan, RSA = RSAcosmos, $G=$ Goto, $D=$ Digistar $/$ Evans\&Sutherland,$d=$ Digitalis 


\section{Les planétariums fixes en France}

Une grande variété caractérise le monde des planétariums, tant pour les équipements que les structures les abritant et les gérant. En effet, les planétariums sont gérés par des collectivités territoriales, par des associations ou par des sociétés d'économie mixte (SEM), et ils fonctionnent soit au sein de centres de diffusion scientifique, ou en autonomie - pour la plupart des petites structures. L'habitude de classer les planétariums selon leur diamètre - ce qui en général correspond à une répartition en fréquentation - a été supplantée par des critères de classification plus techniques, selon le type d'équipement développé. Le tableau ci-contre donne un aperçu de la situation des 26 planétariums fixes ayant une coupole de 5 mètres de diamètre et au-delà.

Divers systèmes de projection équipent les salles, systèmes essentiellement de deux types : optomécaniques et numériques. Une analyse technique des équipements est proposée dans un article ci-après (Lionel Ruiz et Agnès Acker). Les systèmes classiques projettent optiquement un ciel étoilé qui peut être de toute beauté, et sont préférés par les structures installées loin des éclairages polluant des villes. Les systèmes numériques montrent un ciel plus diffus - tel que les citadins peuvent le voir - et présentent le grand avantage de montrer avec le même système, donc en parfaite synchronisation, des animations vidéo pouvant améliorer la dimension pédagogique des spectacles.

L'ensemble des planétariums français reçoit près de 1500000 visiteurs par an, dont environ $10 \%$ (essentiellement des scolaires) fréquentent des planétariums mobiles.

\section{L'Astronomie itinérante}

Une centaine de structures plus petites enrichit le territoire français (voir la répartition des planétariums en France, www.aplf-planetariums.org). Leur action est orientée essentiellement vers les scolaires; ils se rendent dans les écoles, pour lesquelles l'organisation de déplacements extérieurs est de plus en plus difficile, et qui donc ne peuvent visiter que rarement les planétariums fixes. Aussi, plusieurs structures fixes, planétariums ou centres de culture scientifique et technique, élargissent leur action en augmentant leur équipement d'un dispositif itinérant.

Les collectivités peuvent encourager ces activités qui valorisent leur territoire, comme le montre l'exemple de Châteaubriant : début 2005, la ville a fait l'acquisition du petit projecteur avec sa coupole et en a confié la gestion et l'itinérance à l'association Castel-astro créée à cet effet. En relation avec l'inspecteur de l'Éducation nationale, des séances de niveaux différents sont ainsi présentées aux écoles, aux collèges et lycées. En 2005, 14 établissements scolaires ont bénéficié de 40 séances de planétarium, dans un périmètre d'environ 80 kilomètres. Les trois animateurs sont bénévoles ; la ville de Châteaubriant et la communauté de communes prennent en charge les interventions des écoles les concernant : un exemple à suivre par d'autres villes.

D'autres structures itinérantes ne bénéficient d'aucun soutien public, quoique étant très actives et accueillant plus de 10000 visiteurs par an en milieu rural (Montredon-Labessonie dans le Tarn). Ces petites structures sont souvent pénalisées par l'arrivée dans leur voisinage d'un nouveau planétarium prestigieux et très bien médiatisé ; la solution idéale serait que ces très grandes structures citadines travaillent avec les petits planétariums ruraux, en faisant des sortes d'antennes dont ils assureraient la promotion et le fonctionnement.

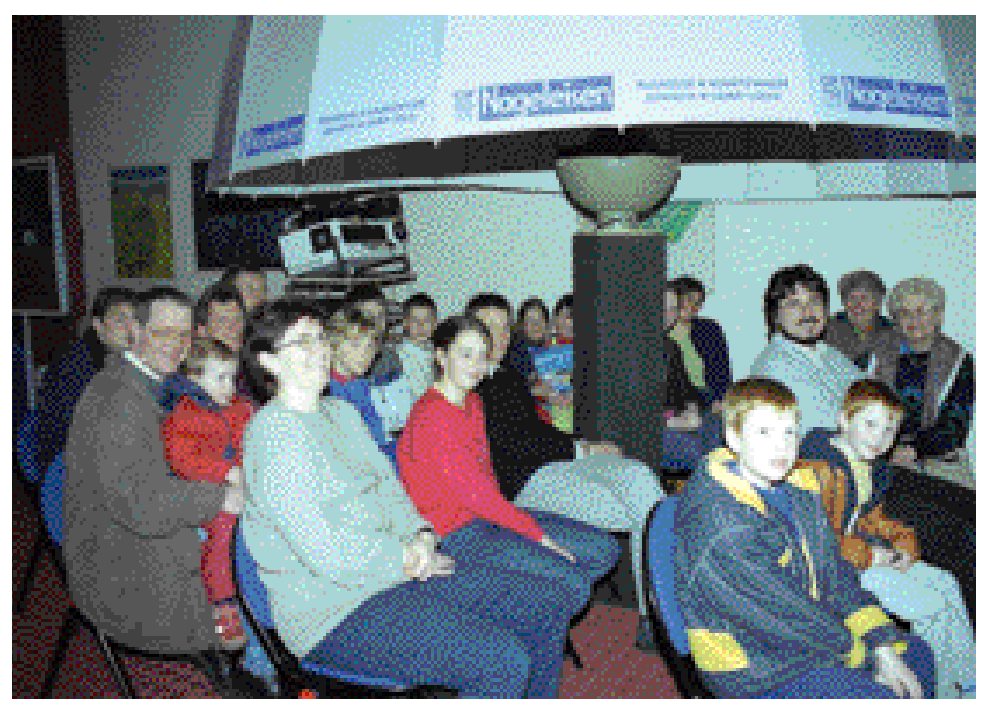

Sous la coupole d'un planétarium itinérant (c) Chasseurs d'éclipses/Kruth

\section{Un domaine porteur ?}

Une réelle dynamique marque la création des planétariums au fil des années : après la grande relance des années 1980-85 (7 créations), le nombre d’implantations croît régulièrement à raison d'une création par an en moyenne. Quelle peut être la place du planétarium, excellent outil de la culture scientifique et technique, dans notre société ? Voici 
quelques témoignages venus de divers types de planétariums, et rapportés dans la revue Planétariums en 2004 :

- "Le planétarium est un équipement culturel (la science, c'est de la culture) dont la spécificité est la thématique astronomique; c'est ce positionnement qui nous permet d'exister dans le champ culturel de l'agglomération et de la région en terme de diffusion des savoirs, de création, de citoyenneté, de communication » (Vaulx-en-Velin);

- "Le planétarium permet une diffusion rapide de l'information, fait rêver, et s'inscrit dans un contexte pluriculturel »(Reims);

- "Le planétarium est en phase avec l'intérêt porté par le public à l'espace, l'actualité astronomique relayée par les médias, la pratique de l'astronomie dans les établissements scolaires » (Épinal) ;

- "Les groupes scolaires sortent difficilement. Le public se désintéresse de la science »(Strasbourg);

- "L'Astronomie a toujours été une discipline très porteuse, et ce d'autant plus que le public n'en connaît que ce que les médias (avec toutes les critiques que l'on peut en faire) veulent bien en dire. Cette discipline n'étant plus enseignée véritablement à l'école, le rôle des planétariums est en ce sens incontournable » (Aixen-Provence) ;

- "Très peu d'offres d'emploi circulent, du fait sans doute de restrictions ou orientations budgétaires. On peut espérer que cela redémarre avec l'ouverture de plusieurs planétariums attendue en 2006 » (Villeneuve d'Ascq) ;

- "Difficile de s'adapter à la culture du zapping, il faut beaucoup d'énergie pour susciter un intérêt. Mais si l'on se limitait à la culture star academy et télé réalité, nous élargirions le fossé entre les élites et la France d'en bas. Alors il ne faut pas baisser les bras » (Tarn).

\section{Créer un planétarium}

Tout nouvel équipement créé avec succès présuppose en général deux conditions :

- l'activité reconnue d'un groupe d'acteurs compétents (souvent réunis en association), solidaires, généreux et passionnés, qui proposent un vrai « porteur de projet »;

- le soutien financier d'une collectivité ou d'une institution ayant compris qu' il n'y a pas de citoyenneté sans partage des savoirs " (Condorcet) - structure contrainte par les contextes politiques, mais qui seule peut apporter l'investissement nécessaire et la garantie d'un fonctionnement pérenne au-delà des personnes.

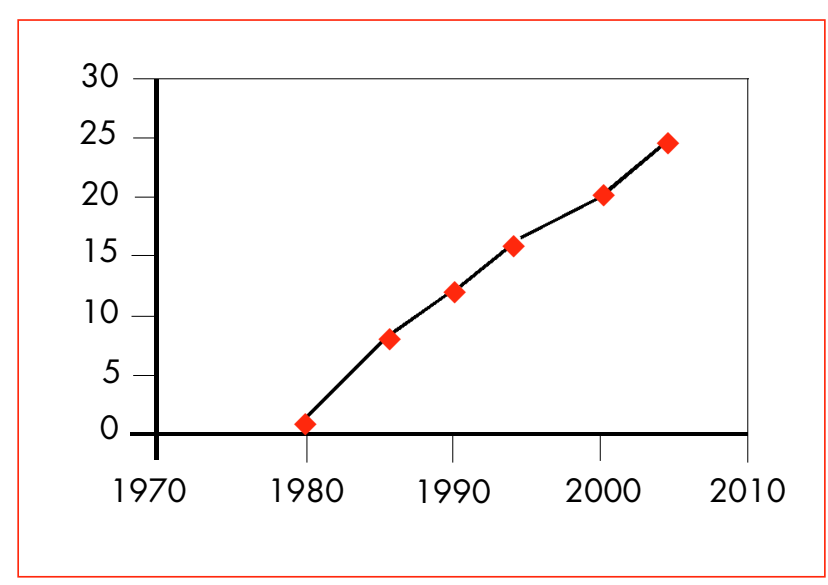

Évolution du nombre de planétariums entre 1981 et 2006

Ce sont deux mondes aux démarches et aux enjeux très différents, et qui pourtant arrivent à co-habiter et œuvrer harmonieusement ensemble. Une aide importante peut être apportée par les services du rectorat, qui parfois détache un enseignant au service des actions pédagogiques menées dans un planétarium. Enfin, tout projet gagne à se coordonner avec les activités des autres sites de diffusion scientifique, et clubs ou sociétés d'Astronomie œuvrant dans la région.

L'APLF apporte soutien et information dans la mesure de ses capacités, pour aider à constituer un dossier à soumettre aux financeurs potentiels, sachant que tout projet est fortement inscrit dans son contexte culturel, économique et social.

\section{Les spectacles de planétariums : attrayants et didactiques}

Deux types de séances sont présentées dans tous les planétariums : spectacles pour le public familial, démonstrations didactiques pour les scolaires. Dans tous les cas, la technique et la créativité se conjuguent pour rendre l'univers accessible ; cet univers est merveilleux, mais il est difficile de montrer sans trop les simplifier ses mécanismes complexes, imaginés et vérifiés grâce à des outils et méthodes développés à travers les grandes étapes de l'histoire des sciences.

Les séances de planétarium classiques sont centrées sur l'animateur, qui montre le ciel étoilé et commente en direct les phénomènes célestes, ajoutant la richesse de sa présence à celle de l'exposé - qui va différer d'un animateur à l'autre. Ce type de séance nécessite un présentateur aimant parler et sachant s'adapter à son public, sans être pédant ou trop réducteur. 
Tous les planétariums présentent ce type de séances « live » à leur public scolaire, et dans l'espace «questions du public ». N'oublions pas que la parole est le premier outil de l'animateur, ce qui lui pose de sévères contraintes et un devoir constant de formation et de mise à niveau.

Pour apporter des éléments visuels complétant les possibilités du projecteur principal, les salles s'équipent de projecteurs supplémentaires : projecteurs de diapositives (Simda relance le marché), vidéoprojecteurs, projecteurs d'effets spéciaux (rotation d'une planète, passage de nuages, survol d'un paysage de planète, passage de comète...). Ces effets doivent être synchronisés avec le discours, donc il est nécessaire que l'animateur soit aidé par un collègue, ou bien que l'ensemble soit pré-enregistré.

Adapter une salle à un tel spectacle prend du temps avec un projecteur optomécanique. Aussi, depuis plusieurs années, le système de projection numérique est privilégié, une grande quantité de spectacles pouvant se dérouler en parfaite synchronisation, sans nécessiter l'achat et l'installation de projecteurs annexes. De plus, les possibilités de ce système permettent de dépasser le seul cadre de l'Astronomie, pour une ouverture du planétarium vers d'autres sciences et le moyen d'attirer de nouveaux publics. Bien entendu, ces systèmes permettent de passer au contrôle manuel de l'appareil pour que l'animateur puisse interagir en direct avec le public. L'animateur se voit donc promu à un double rôle : celui de spécialiste d'une technique de pointe, et celui de conférencier.

\section{Du côté du public : le témoignage du planétarium de Marseille}

Le planétarium Andromède est récemment passé au numérique, et réalise avec brio ses propres spectacles. Lionel Ruiz témoigne :

"Avec un système optomécanique, passé le moment d'extase et d'acclamations où la nuit étoilée apparaît, la séance reprend avec ses explications et une certaine démystification de son contenu jusqu'à une fin sans artifice où les lumières de la salle réapparaissent.

Avec un système numérique, le ciel étoilé n'est qu'un des points forts de la séance. L'attention est sans cesse sollicitée par des effets qui recaptivent l'audience avec une facilité déconcertante, par exemple en quittant le plan de l'écliptique pour expliquer pourquoi les planètes apparaissent alignées dans le ciel, ou en plongeant dans les anneaux de Saturne, ou en revivant la formation du système solaire en pleine voûte, en zoomant sur une nébuleuse ou une galaxie, et en quittant le plan

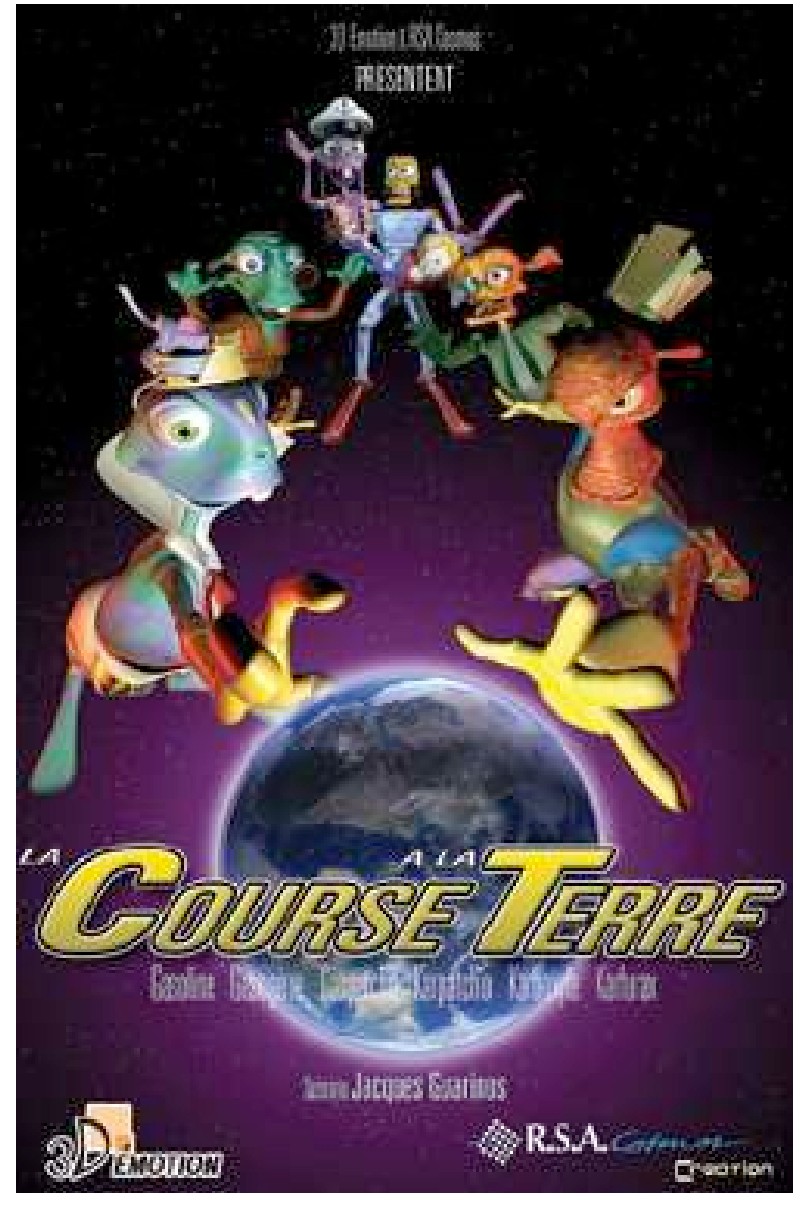

La course à la Terre, spectacle du planétarium de Saint-Étienne (c) RSA Cosmos

galactique pour montrer la forme spiralée de notre tourbillon stellaire, puis en visionnant la répartition des galaxies en couleur sur le fond stellaire omniprésent, jusqu'au moment où l'astre du jour, le Soleil, réapparaît sur la coupole du planétarium, éblouissant, sur un fond de ciel revenant graduellement jusqu'au bleu.

Quand les spectateurs ressortent, ils ont peut-être oublié à quel point il était beau de voir le firmament étoilé bien contrasté, mais ils ont l'impression d'avoir vécu beaucoup de choses crescendo. Et nous, les animateurs, sommes les premiers touchés d'être à ce point envahis par l'émotion toute vivace qu'ils nous témoignent en sortant du dôme ».

\section{Des spectacles co-produits}

Les grands planétariums, disposant de moyens importants, présentent des spectacles construits avec des prestataires extérieurs, professionnels de l'image et du son. Le coût de la conception, de la réalisation, et de la production de ces spectacles est conséquent : le thème et les éléments du spectacle 
sont proposés par le responsable du planétarium veillant à la qualité et la rigueur du contenu astronomique - puis un scénariste professionnel écrit le texte et le story-board ; une musique avec des effets spéciaux est créée pour ponctuer les divers actes du spectacle ; des acteurs professionnels disent le texte, enregistré dans un studio en synchronisation avec la musique ; enfin, des images et séquences vidéos sont collectées et créées, pour illustrer le spectacle tant pour en augmenter l'attractivité que pour le rendre plus pédagogique. L'ensemble peut être réuni sur un support unique à la demande des petites structures. Notons que l'arrivée des planétariums numériques avec vidéo pleine voûte représente un stade supérieur de technicité, et demande un travail et un coût bien plus importants (plusieurs milliers d'euros).

Les spectacles enregistrés sont assez facilement adaptables à la majorité des salles numériques, ils sont donc généralement créés et produits en collaboration par plusieurs planétariums, et des échanges se font à travers le réseau APLF. Les spectacles pour scolaires ont ainsi été enrichis de superbes productions créées par le planétarium Galilée de Montpellier, ceux du Palais de l'Univers à Cappelle-la-Grande et du Forum départemental des Sciences à Villeneuve d'Ascq ou de l'Astronef de Saint-Étienne...

Acheter un spectacle clés en mains diminue les coûts et réduit le travail de l'équipe du planétarium (allant de une à 10 personnes) ; mais cela prive les animateurs de leur « voix » et du bonheur de la création (risquant donc de les démobiliser), et de plus peut conduire à une certaine uniformisation des spectacles en France, alors que chaque structure tient heureusement à conserver son originalité.

\section{Valoriser la recherche par des spectacles à portée européenne}

Les planétariums veillent à présenter au public des connaissances exactes, ainsi que les méthodes et instruments pour les acquérir. Ils participent de ce fait à la valorisation de la recherche qui se construit, qui étend et précise continuellement notre vision de l'Univers. Ces spectacles sont écrits en étroite collaboration avec des chercheurs, et peuvent ainsi bénéficier de remarquables simulations numériques créées dans les observatoires professionnels français et américains (où cette activité est bien plus encouragée qu'en France). Dans certaines circonstances, ces productions sont soutenues financièrement par des institutions scientifiques, souhaitant faire connaître leurs découvertes et innovations technologiques au plus grand nombre.
En partenariat avec des institutions publiques, le réseau de l'APLF a permis de produire des spectacles en six langues et de les diffuser au niveau européen, dans une quarantaine de planétariums. Les spectacles sont déclinés en versions adaptées au niveau technique de l'environnement audio-visuel de chaque planétarium : niveau 1 (projecteur de diapositives), niveau 2 (vidéoprojecteur en plus), niveau 3 (projection pleine voûte ou/et panoramique en plus).

Le CNES (centre national d'Études spatiales) a été le commanditaire et partenaire de l'APLF en 20012002 pour le spectacle La planète aux mille regards qui permettait au public de mieux connaître les diverses facettes de la planète grâce aux satellites qui l'observent pour la protéger.

L'European Southern Observatory s'est associé à l'APLF en 2002-2003 pour le spectacle Les mystères du ciel austral célébrant le 40 anniversaire de l'ESO. Ce spectacle, créé en forte interaction avec les planétariums allemands, explique clairement les défis technologiques (les grandioses Very Large Telescope, l'optique active et adaptative...) permettant de sonder les zones poussiéreuses de formation stellaire, d'étudier les phases finales de la vie stellaire, de détecter les lointaines galaxies, et de présenter les grands enjeux de la recherche actuelle (problèmes de la matière et de l'énergie « noires »). En particulier, une simulation vidéo de l'expansion de l'Univers a été présentée, en évitant le trop classique piège d'une « explosion primordiale ».

Enfin, en 2003, le spectacle Vémus et les chasseurs de planètes a été créé par l'APLF à l'occasion du passage de Vénus devant le Soleil : les aventures historiques de l'observation de cet évènement ont été contées, les aspects de Vénus ont été présentés, et les techniques pour découvrir les exoplanètes ont été explicitées.

Célébrer les grands évènements cosmiques en relation avec de grandes institutions scientifiques, est extrêmement gratifiant pour les planétariums. De plus, faire découvrir au public - et particulièrement aux jeunes les beautés et les enjeux de la recherche scientifique, contribue à orienter leur devenir professionnel vers des branches techniques de l'économie nationale - qui sont actuellement délaissées - et à favoriser l'émergence de nouvelles générations de chercheurs.

\section{Quand I'art et la science se donnent la main}

Les planétariums racontent avec talent les découvertes des astrophysiciens, découvertes qui étonnent et enchantent les spectateurs, et souvent les 
transportent bien loin de leurs soucis et grisailles. Au-delà des différences de races et de cultures, l'Astronomie apporte à chacun la dimension suprême de son environnement, le plongeant dans des espaces démesurés et dans les tempêtes d'une histoire cosmique nous affiliant aux étoiles. Le regard des artistes ne peut rester indifférent : danseurs, musiciens, poètes, peintres, écrivains, célèbrent avec les scientifiques la beauté, la violence, la complexité du cosmos.

Depuis 2002, l'Observatoire de l'Espace du CNES conçoit, de concert avec l'APLF, une politique de manifestations de culture scientifique et technique spatiale. Mots, contes, récits, et images de l'Espace, est une alliance du vocabulaire, de l'imaginaire et de l'Espace. Grâce à ce partenariat actif, chaque année, des dizaines de planétariums célèbrent la constellation de 10 mots proposés lors de la Semaine de la Francophonie, sous l'égide du ministère de la Culture. Une opération qui ravive les liens des structures avec la direction régionale des Affaires culturelles et renforce leur propre rôle dans la cité.
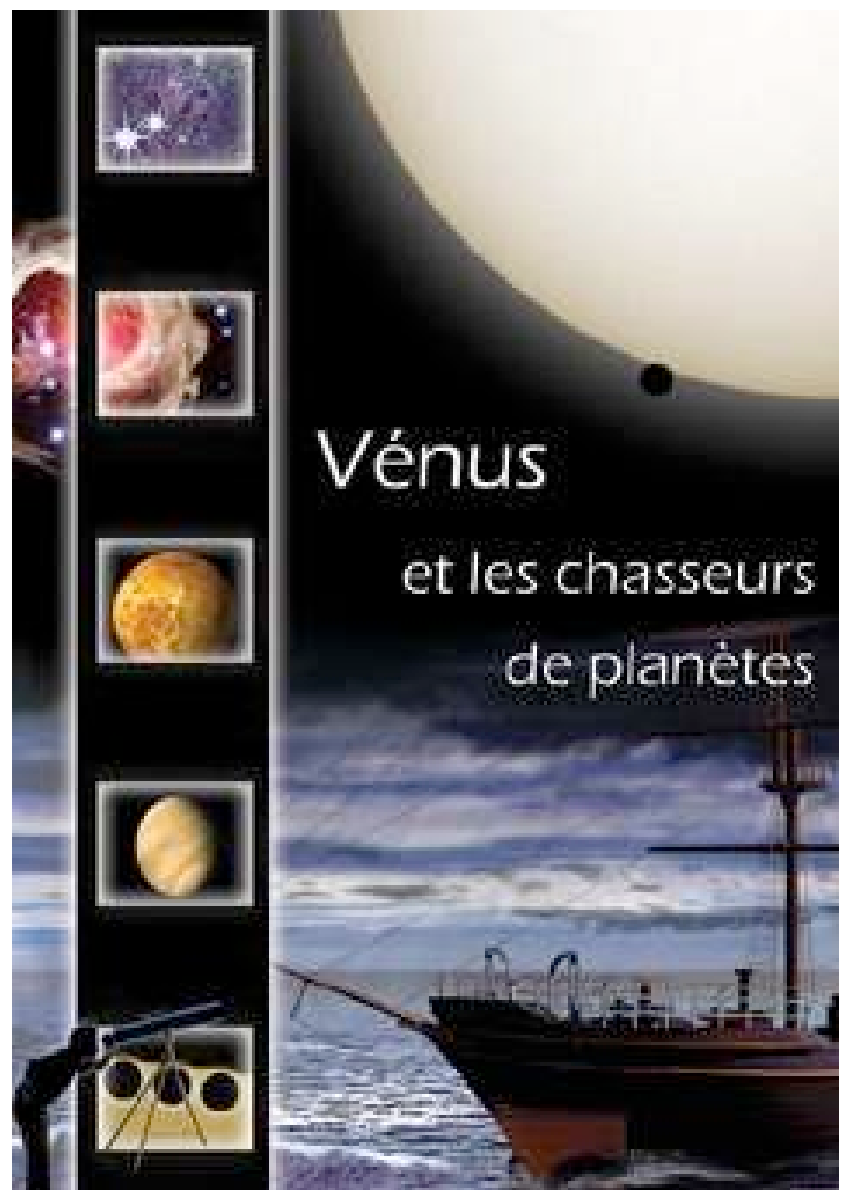
de planètes

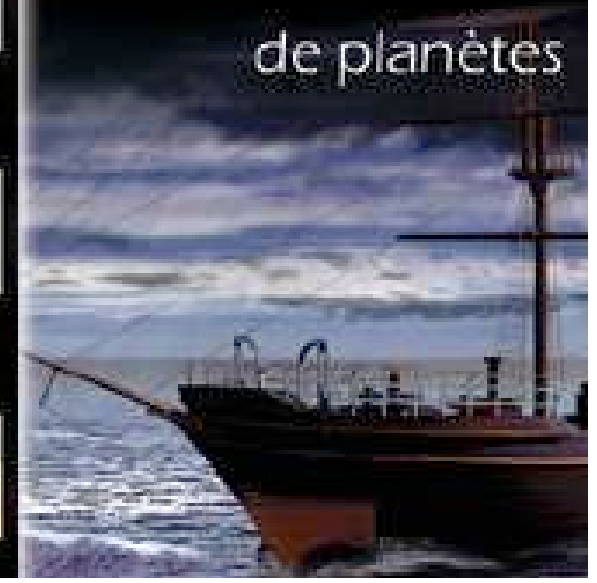

(C) APLF
Des langages communs ont été émulés à l'Astronef de Saint-Étienne dès 2003 en relation avec la Compagnie ART'M. Des métiers et des cultures différents sont mis en contact : astrophysiciens du CRALCNRS de Lyon, gens de l'image et spécialistes du planétarium, professionnels de théâtre, de l'écriture et de film, qui ensemble ont pu réussir le projet Vibrato instructif autant que magique et rencontrant un écho évident auprès des publics, jeunes des collèges et lycées, et adultes.

Au centre astronomique de Saint-Michel-l'Observatoire, des acteurs du Théâtre Libre font résonner dans le planétarium les voix de Einstein et Pécuchet au pays des neutrinos. À Reims, des musiques nouvelles se sont exprimées dans le cadre d'un spectacle spécifique. À Strasbourg, la compagnie Articulations a réalisé un spectacle dédié au Ciel des poètes, et la Fête de la Musique a réuni un orchestre de jeunes musiciens, violons et flûtes, ciselant leurs harmonies sous la voûte étoilée.

Un ciel qui étonne et qui fait rêver : traquer l'inconnu est une préoccupation commune aux scientifiques et aux poètes, une double identité dans laquelle se reconnaît chaque planétarium.

\section{Les animateurs, médiateurs scientifiques}

Armé d'une solide culture astronomique, apte à enthousiasmer un public, acteur, parfois clown, c'est le profil culturel moyen d'un animateur classique de planétarium. L'animateur doit s'adapter à son public, comme en témoigne Elie Bosc qui a expérimenté plusieurs types de structures :

"Dans un petit planétarium de type club Astro privé, l'on s'adresse généralement à ses camarades et à des visiteurs qui ont déjà quelques connaissances. Là, le planétarium prend la fonction d'un forum, on va traiter de sujets particuliers, les anciens vont expliquer aux nouveaux les bases de la géométrie du système solaire, préciser la position de constellations que l'on évoque rarement, faire le point sur la position des planètes, préparer les observations du soir en indiquant où se trouve tel ou tel objet Messier... Dans un planétarium public, les visiteurs sont en grande majorité des personnes individuelles volontaires (adultes, familles) ou des groupes (scolaires, vacanciers) plus captifs, qui n'ont pas forcément choisi de venir : c'est le professeur ou le moniteur qui a choisi pour eux. Dans ce cas, la proportion de personnes ayant des connaissances en Astronomie est très minoritaire, ce dont l'animateur doit tenir compte. La plupart du temps les individuels viennent un peu au hasard, au même titre qu'ils visitent un musée, un château ou un spectacle. Cela 


\section{Animateur de planétarium : un besoin de formation}

«Animateur de planétarium » : ce métier exigeant de multiples capacités et savoir-faire doit avoir une place reconnue dans notre société. Une formation spécifique et institutionnalisée doit être offerte à ces animateurs qui œuvrent aux frontières des établissements scolaires. Ces métiers nouveaux souffrent de n'être pas encore reconnus officiellement dans le domaine de l'économie sociale. Ils devraient être validés, idéalement par les ministères conjugués : Éducation nationale et Recherche, et Jeunesse et Sport.

\section{Brevet d'État d'Animateur Technicien de l'Éducation populaire et de la jeunesse}

Lutter contre le désintérêt des jeunes pour les disciplines scientifiques, apporter aux animateurs de planétariums et de clubs d'Astronomie des bases solides tant dans le domaine de l'Astronomie que dans ceux de la pédagogie et de la gestion d'une structure, tels sont les objectifs qui ont présidé à la mise en place du BEATEP, spécialisation « activité scientifique et technique, option Astronomie »

Le BEATEP est le diplôme reconnu pour organiser des activités de loisirs, de prévention ou d'insertion sociale, pour encadrer des activités d'animation, d'éducation populaire en matière d'environnement, de patrimoine culturel, de loisirs scientifiques et techniques... Il permet d'avoir accès aux emplois dans les structures d'animation, de loisirs, de centres de vacances, des associations d'éducation populaire. Le BEATEP permet par ailleurs de se présenter au concours d'animateur de la fonction publique territoriale. Le BEATEP a été mis en place en 2006 autour de l'« activité
Scientifique et Technique »; il s'organise autour de l'Astronomie, car c'est la science qui rassemble et véhicule de façon attractive de nombreuses disciplines: Physique, Chimie, Géologie, Biologie... permettant de porter un regard nouveau sur notre environnement à grande échelle, l'Univers, et de comprendre ses mécanismes tout en admirant ses merveilles : la passionnante mission des planétariums.

\section{Le contenu et l'organisation de la formation}

Des connaissances et des compétences multiples sont fournies par une constellation innovante de formateurs reconnus. Les formateurs en Astronomie relèvent du ministère de l'Éducation nationale et de la Recherche, et le Brevet est délivré par le ministère de la Jeunesse et des Sports.

Initiée par l'APLF, la formation est portée par l'organisme agréé CEMEA Alsace (centres d'entraînement aux méthodes d'éducation active, www. cemea.asso.fr). Les formateurs en Astronomie appartiennent aux Observatoires de Paris et de Strasbourg (www.obspm.fr et http://astro.ustrasbg.fr). Toute la formation couvre 14 semaines, dont six assurées par les formateurs CEMEA/Alsace. Afin d'assurer une efficacité maximale (sans perte de temps en déplacements), la formation se passe complètement « en résidence ». Six semaines sont consacrées aux unités de formation générale et pédagogique, et se déroulent dans les locaux du CREPS de Strasbourg. Les 240 heures dédiées à l'Astronomie et aux pratiques pédagogiques spécifiques couvrent huit semaines, dont deux (en juillet 2006) à Saint-Michel-l'Observatoire : l'une à l'Observatoire de Haute
Provence (OHP) et l'autre au Centre d'Astronomie. Les six autres semaines se déroulent à Marly-le-Roy, dans les locaux de l'INJEP, la formation de l'une de ces semaines se passant à l'Observatoire de Meudon lui-même. De nombreux enseignants-chercheurs de l'Observatoire de ParisMeudon apportent leur contribution, sous la tutelle du président de l'Observatoire, Daniel Egret, et la coordination de Chantal Balkowski, chercheurs issus de divers instituts (institut de Mécanique céleste et Calcul des Éphémérides, unité de formation et d'enseignement de l'Observatoire de Paris, institut d'Astrophysique de Paris). Une semaine est animée par les astronomes de Strasbourg, coordonnés par Agnès Acker, avec la contribution de RSA Cosmos, constructeurs français de planétariums. Les détails et le calendrier des enseignements sont indiqués sur le site : www.obspm.fr/ $\sim$ webufe/beatep/

\section{Et après le BEATEP ?}

Le BEATEP est valable jusqu'en 2009, mais depuis 2006 il est remplacé par le BPJEPS (Brevet Professionnel, niveau 4, bac) qui ne propose pas une option « Animation scientifique ». L'option « Loisir tout public » permettrait de créer un certificat de spécialité, unité complémentaire en activité scientifique et technique, avec environ 100 à 200 heures d'animation en Astronomie. Le BESJEPS (Brevet d'État Supérieur, niveau 3 , bac +2 , pré-requis $=$ expérience et niveau BAC) est actuellement en préparation par l'APLF, ses partenaires de l'Astronomie professionnelle et les organismes nationaux spécialistes de la formation. 


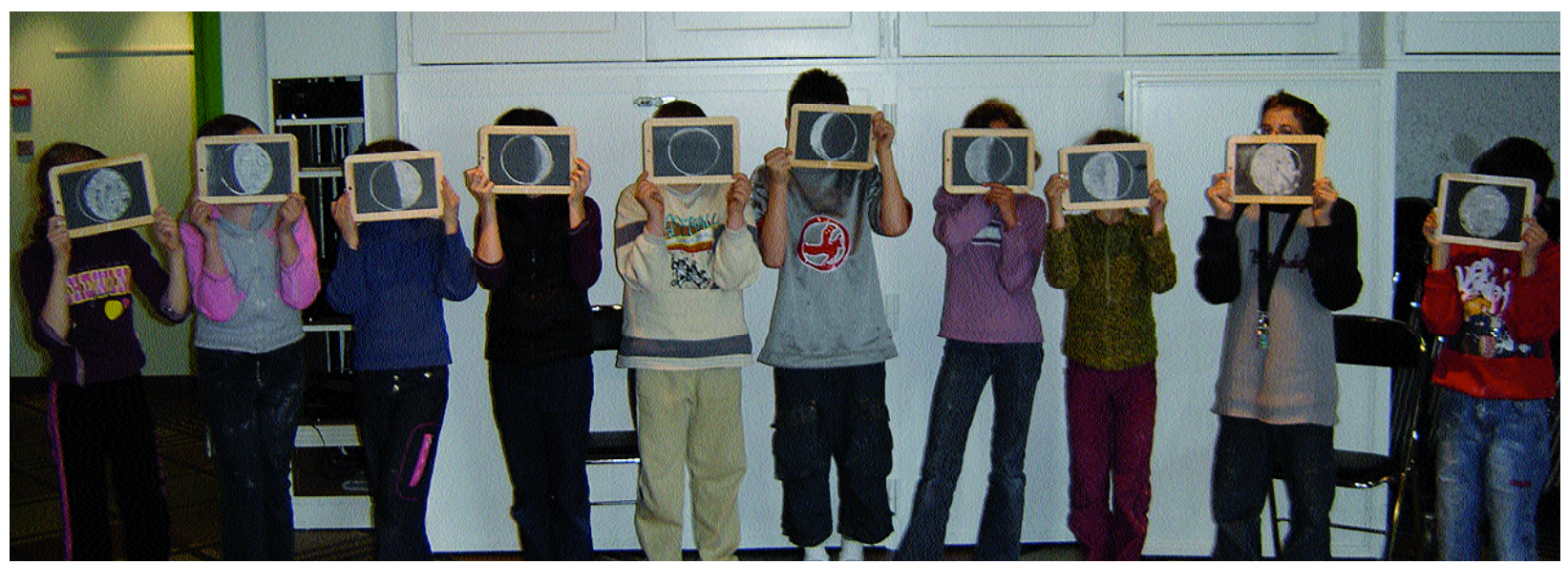

Une animation organisée autour des phases de la Lune au planétarium d'Épinal. (c) Didier Mathieu

participe d'une volonté globale de se cultiver mais surtout de passer un bon moment, de se divertir intelligemment. »

Mais l'animateur doit de plus savoir maîtriser une technique parfois complexe (malgré les louables efforts des constructeurs de planétariums), être capable de construire un spectacle et avoir le temps de le réaliser. Calculer en projection sphérique une scène ou la filmer requiert (outre le matériel et une bonne formation dans le domaine), une importante durée de développement et de montage. L'animateur/programmateur devient parfois infographiste expert en 3DS Max, suite Adobe et Photoshop... et doit faire preuve d'imagination pour cuisiner tout ces ingrédients dans la programmation. Une reconsidération des besoins en postes est souvent nécessaire lors du passage au numérique. Enfin, la représentation des phénomènes cosmiques au planétarium doit être complétée par des expériences et démonstrations menées dans des locaux annexes : les expertises de l'animateur moderne sont diverses et de haut niveau.

\section{Les métiers des planétariums}

La revue Planétariums rapporte en 2004 les résultats d'une enquête menée auprès des planétariums fixes. Environ 150 personnes salariées sont à l'œuvre dans 20 planétariums, dont environ les trois quarts à temps plein, le reste étant composé de mi-temps, vacataires... Il s'agit essentiellement d'animateurs scientifiques $(31 \%)$, travaillant avec des techniciens ou/et informaticiens (19\%), à côté de personnels assurant l'accueil ou gérant la boutique (18\%); les responsables $(15 \%)$ sont assistés de secrétaires $(8 \%)$, de chargés de communication $(5 \%)$, et de personnel chargé de l'entretien des locaux et de la sécurité (4\%) - ces derniers postes n'étant présents que dans les structures importantes. Nous constatons l'arrivée des universitaires, docteurs en Astrophysique, à des postes de responsabilité.

Environ $65 \%$ des emplois sont stables, les autres étant de type CDD, CES, saisonniers, emplois-jeunes. Le salaire le plus bas est de l'ordre du salaire minimum, et le plus élevé est supérieur à 3000 euros brut, mais avec une grande disparité d'un site à l'autre. À cet égard, il semble qu'œuvrer dans des structures publiques serait un peu plus avantageux. Lors des recrutements, les candidats sont évalués d'après leurs compétences pédagogiques et connaissances scientifiques, leur aisance face au public, leur disponibilité en fin de semaine et les jours fériés - ce dernier point représentant l'inconvénient majeur du métier (à côté d'une certaine répétitivité et de salaires trop bas). Mais les animateurs sont unanimes à apprécier la richesse du contact avec le public, la diversité des activités, le contact avec la recherche et l'actualité scientifique.

\section{En guise de conclusion}

Enrichi par les apports de la télévision, du cinéma, des salles IMAX, le planétarium d'aujourd'hui visualise l'Univers en 3D, à travers les images transmises par les VLT et HST, mais aussi les images de la Biologie, de la Chimie... toutes images issues de la recherche actuelle. Ce " théâtre pour toutes les sciences " peut délivrer un passeport pour l'Univers, du microcosme à l'immense espace astronomique.

Les planétariums participent à l'éducation des jeunes, ils valorisent la recherche scientifique, ils renforcent la cohésion sociale, ils s'inscrivent parfaitement dans les préoccupations des collectivités territoriales. 